\title{
Adición de aceites vegetales a la dieta de cabras lecheras: efecto sobre el contenido de ácidos grasos de la grasa láctea
}

\author{
Adding plant oils to dairy goat diets: effect on fatty acid content of milk fat \\ AL Martínez Marín ${ }^{\text {a* }}$ P Gómez-Cortés ${ }^{\mathrm{b}}$, LM Pérez Alba ${ }^{\mathrm{a}}$, M Juárez ${ }^{\mathrm{b}}$, AG Gómez Castro ${ }^{\mathrm{a}}$, \\ M Pérez Hernández ${ }^{\mathrm{a}}$, MA de la Fuente ${ }^{\mathrm{b}}$
}

a'Departamento de Producción Animal, Universidad de Córdoba, España.

'Instituto de Investigación en Ciencias de la Alimentación (CSIC-UAM), Universidad Autónoma de Madrid, España.

\begin{abstract}
SUMMARY
The aim of this study was to investigate the effects of adding differently unsaturated vegetable oils to a dairy goat diet on fatty acid content of milk fat. Twelve Malagueña goats were randomly allocated to one of four treatments: no oil basal diet and the same basal diet supplemented with 48 $\mathrm{g} / \mathrm{d}$ of high oleic sunflower oil, regular sunflower oil or linseed oil. The basal diet was made of alfalfa hay and a pelleted concentrate (30:70 w/w). The concentrate included the respective oils. Supplemented diets decreased medium chain saturated fatty acid content in milk fat $(\mathrm{P}<0.05)$. High oleic sunflower oil increased stearic and oleic acid contents $(\mathrm{P}<0.05)$ and did not affect neither the content of trans monounsaturated fatty acids of 18 carbon atoms nor conjugated linoleic acid content $(\mathrm{P}>0.05)$. Regular sunflower oil increased $\mathrm{C} 18: 1 \mathrm{t} 10$, vaccenic and rumenic acid contents as well as linoleic to $\alpha$-linolenic acid ratio $(\mathrm{P}<0.05)$. Linseed oil increased vaccenic, rumenic and $\alpha$-linolenic acid contents and substantially decreased linoleic to $\alpha$-linolenic acid ratio $(\mathrm{P}<0.05)$ without affecting $\mathrm{C} 18: 1 \mathrm{t} 10$ content $(\mathrm{P}>0.05)$. It was concluded that compared to high oleic o regular sunflower oils, adding linseed oil in dairy goat diets modifies milk fat fatty acid content more favourably from the point of view of human health.
\end{abstract}

Key words: plant oil, dairy goats, fatty acid profile, milk fat.

\section{RESUMEN}

El objetivo del presente trabajo fue investigar el efecto de la adición de aceites vegetales de diferente grado de insaturación a la dieta de cabras lecheras sobre el perfil de ácidos grasos de la grasa láctea. Doce cabras de raza Malagueña fueron asignadas al azar a uno de cuatro tratamientos: dieta basal sin aceite añadido y la misma dieta basal adicionada con $48 \mathrm{~g} / \mathrm{d}$ de aceite de girasol alto oleico, aceite de girasol normal o aceite de lino. La dieta estuvo compuesta por heno de alfalfa (30\%) y un concentrado granulado (70\%) en el que se incluyó el aceite correspondiente. Todos los aceites redujeron el contenido de ácidos grasos saturados de cadena media de la grasa láctea $(\mathrm{P}<0,05)$. El aceite de girasol alto oleico aumentó el contenido de los ácidos esteárico y oleico $(\mathrm{P}<0,05)$ y no afectó al de los ácidos grasos monoinsaturados trans de 18 átomos de carbono ni al de ácido ruménico $(\mathrm{P}>0,05)$. El aceite de girasol normal incrementó el contenido de los ácidos C18:1t10, vaccénico y ruménico y aumentó el valor de la ratio linoleico/ $\alpha$ linolénico $(\mathrm{P}<0,05)$. Por último, el aceite de lino aumentó el contenido de los ácidos vaccénico y ruménico y redujo el valor de la ratio linoleico/ $\alpha$ linolénico $(\mathrm{P}<0,05)$ sin afectar al contenido de C18:1t10 $(\mathrm{P}>0,05)$. Los resultados obtenidos permiten concluir que la adición de aceite de lino a la dieta de cabras en lactación, en comparación con la de los aceites de girasol alto oleico y normal, modifica el perfil de ácidos grasos de la grasa láctea en un sentido más favorable desde el punto de vista de la salud humana.

Palabras clave: aceite vegetal, cabras lecheras, perfil de ácidos grasos, grasa láctea.

\section{INTRODUCCIÓN}

Una de las principales razones para incluir fuentes de grasa en la dieta de los rumiantes es la posibilidad de modificar el perfil de ácidos grasos de la grasa láctea, debido al interés creciente de los consumidores en el efecto que la grasa consumida tiene sobre la salud y la prevención de ciertas enfermedades. La inclusión de fuentes de grasa ricas en ácidos grasos insaturados (AGI) en la dieta de

Aceptado: 04.07.2013.

* Campus Universitario de Rabanales, Carretera Madrid-Cádiz, km. 396, 14014 Córdoba, España; pa1martm@uco.es las hembras rumiantes lecheras, ha demostrado ser una estrategia muy efectiva para modificar el contenido de ciertos ácidos grasos de la grasa láctea (Chilliard y col 2007). Estos cambios pueden ser considerados favorables desde el punto de vista de la salud humana como la reducción del contenido de ácidos grasos saturados (AGS) de cadena media (Ulbricht y Southgate 1991), la disminución de la relación entre los ácidos grasos poliinsaturados (AGPI) de las series n-6 y n-3, representadas mayoritariamente por $\mathrm{C} 18: 2 \mathrm{c} 9 \mathrm{c} 12$ (ácido linoleico) y C18:3c9c12c15 (ácido $\alpha$-linolénico) (Simopoulos 2008), y el incremento de C18:1t11 (ácido vaccénico, Field y col 2009) y de C18:2c9t11 (ácido ruménico, Benjamin y 
Spener 2009). Sin embargo, la adición de AGI a la dieta de las hembras rumiantes también puede aumentar el contenido de C18:1t10 de la grasa láctea, lo que es contraproducente porque su consumo se ha asociado con el riesgo de aterosclerosis (Bauchart y col 2007).

La mayoría de los trabajos de investigación que han estudiado el efecto de la inclusión de aceites vegetales ricos en AGI sobre el perfil de ácidos grasos de la grasa láctea de cabras se han llevado a cabo en razas de elevado potencial productivo (Chilliard y col 2007). Sin embargo, existen muy pocos estudios en la literatura que hayan investigado el efecto de la inclusión de aceites vegetales en la dieta sobre un elevado número de ácidos grasos de la grasa láctea con cabras de razas autóctonas (Luna y col 2008, Martínez Marín y col 2011). En estos y otros trabajos publicados (Bernard y col 2005, Mele y col 2008, Bernard y col 2009a), los aceites utilizados han sido los de girasol alto oleico, soja y girasol, y lino que son ricos en los ácidos oleico (C18:1c9, 80\%), linoleico (54 y $63 \%$ ) y $\alpha$-linolénico (54\%), respectivamente. Los aceites de soja y girasol aportan cantidades apreciables de ácido oleico (22\%) y el aceite de lino aporta cantidades similares de los ácidos oleico y linoleico (16 y 19\%) siendo el único con una relación linoleico/ $\alpha$-linolénico (n-6/n-3) menor que cero (FEDNA 2010). Los resultados obtenidos indican que los efectos observados sobre el contenido de ácidos ácidos grasos de la leche son principalmente dependientes del ácido graso mayoritario en los aceites probados (Bernard y col 2005, Maia y col 2006, Luna y col 2008, Martínez Marín y col 2011), pero también influye la cantidad de aceite aportado (Martínez Marín y col 2012), la proporción de forraje en la dieta (Mele y col 2008) y el modo de conservación del forraje (Bernard y col 2009a).

El objetivo del presente trabajo fue investigar el efecto de incluir aceites vegetales con diferente grado de insaturación en la dieta de cabras lecheras sobre el perfil de ácidos grasos de la grasa láctea.

\section{MATERIAL Y MÉTODOS}

Todas las experiencias se llevaron a cabo en el Centro Usuario de Animales de Experimentación CO/5/U ubicado en el edificio del Departamento de Producción Animal de la Universidad de Córdoba. Se utilizaron 12 cabras de raza Malagueña ( $45 \pm 5$ días de lactación y 47,2 $\pm 4,2 \mathrm{~kg}$ de peso vivo inicial) alojadas individualmente en jaulas de $1,0 \times 1,4 \mathrm{~m}$ con suelo permeable a los excrementos y dotadas de comederos y bebederos independientes. El ordeño se realizó individualmente, una vez al día, a las 8:30 horas utilizando el sistema mecánico instalado en el alojamiento (DeLaval, Madrid, España). La ración se preparó diariamente para cada una de las cabras y se repartió en dos comidas iguales a las 9:30 y 16:00 $\mathrm{h}$.

Durante las semanas previas al comienzo del ensayo, los animales recibieron una dieta común de uso general consistente en granos de cereales y leguminosas, heno de alfalfa y corrector vitamínico mineral. El día previo al comienzo del ensayo se tomaron muestras de leche de cada uno de los animales para analizar los contenidos preexperimentales de ácidos grasos (covariables). Entonces se procedió a asignarlos al azar a uno de cuatro tratamientos (cuadro 1): sin aceite añadido a la dieta basal (CONTROL), y dieta basal adicionada con $48 \mathrm{~g} / \mathrm{d}$ de aceite de girasol alto oleico, girasol normal o lino (dietas GAO, GN y LIN, respectivamente). La dieta basal estuvo compuesta por heno de alfalfa (30\%) y un concentrado granulado (70\%) en el que se incluyeron los aceites. Transcurridos los 21 días del ensayo se tomaron nuevamente muestras de leche para el análisis de ácidos grasos.

\section{ANÁLISIS DE LOS ÁCIDOS GRASOS DE LA GRASA LÁCTEA}

La extracción de la grasa se realizó de acuerdo con el procedimiento descrito por Luna y col (2005). Las muestras de leche fueron atemperadas a $20{ }^{\circ} \mathrm{C}$ y sometidas a un proceso de doble centrifugación, primero en una centrífuga Beckman Avanti 30 (Beckman, Fullerton, USA) y después en una microcentrífuga Eppendorf 5415D (Eppendorf, Hamburgo, Alemania). La primera centrifugación se realizó a una velocidad de $17800 \mathrm{~g}$ durante $30 \mathrm{~min}$ para separar la nata del resto de componentes de la leche. Dicha nata se introdujo en microtubos de centrífuga de $2 \mathrm{ml}$ que se centrifugaron a $19.300 \mathrm{~g}$ durante $20 \mathrm{~min}$ para separar la grasa de la fracción no grasa. La grasa fue extraída con una micropipeta y se depositó en viales de cristal que fueron sometidos a un flujo de nitrógeno, sellados y conservados a $-20{ }^{\circ} \mathrm{C}$ hasta el análisis.

Los ésteres metílicos se prepararon mediante metanólisis básica de la grasa usando una disolución de $\mathrm{KOH}$ en metanol (ISO-IDF 2002a). Para su análisis se utilizó un cromatógrafo de gases (Agilent 6890 N Network System, Palo Alto, USA) equipado con autoinyector y detector de ionización de llama. El perfil de ácidos grasos se determinó de acuerdo con el método descrito por Luna y col (2008). La inyección de las muestras de ésteres metílicos en hexano (1/100) se llevó a cabo en una columna capilar de sílice fundida CP-Sil 88 de $100 \mathrm{~m}$ de longitud, 0,25 $\mathrm{mm}$ de diámetro interno y $0,20 \mathrm{~mm}$ de grosor de la fase estacionaria (Varian, Middelburg, Holanda). El gas portador (helio) operó a una presión constante de $236 \mathrm{kPa}$ y la temperatura del inyector y del detector fue $250{ }^{\circ} \mathrm{C}$. La temperatura inicial del horno fue $160^{\circ} \mathrm{C}$ durante $80 \mathrm{~min}$, después se elevó $10{ }^{\circ} \mathrm{C} / \mathrm{min}$ hasta $210^{\circ} \mathrm{C}$ y se mantuvo constante durante $35 \mathrm{~min}$. La cuantificación de los ésteres metílicos de ácidos grasos individuales se realizó con referencia a una grasa láctea de composición certificada (CRM 164; European Community Bureau of Reference, Brussels, Belgium) de acuerdo con la norma ISO-IDF $\left(2002^{b}\right)$. 
Cuadro 1. Dietas experimentales.

Experimental diets.

\begin{tabular}{|c|c|c|c|c|}
\hline & \multicolumn{4}{|c|}{ Tratamientos $^{1}$} \\
\hline & CONTROL $^{2}$ & GAO & GN & LIN \\
\hline \multicolumn{5}{|l|}{ Dieta, $g / d$} \\
\hline Heno de alfalfa & 600 & 600 & 600 & 600 \\
\hline Concentrado & 1.200 & 1.200 & 1.200 & 1.200 \\
\hline Aceite de girasol alto oleico ${ }^{3}$ & - & 48 & - & - \\
\hline Aceite de girasol normal ${ }^{3}$ & - & - & 48 & - \\
\hline Aceite de lino ${ }^{3}$ & - & - & - & 48 \\
\hline \multicolumn{5}{|l|}{ Composición química } \\
\hline Materia seca, $\%$ & 90,6 & 90,5 & 91,0 & 91,2 \\
\hline Proteína bruta, \% MS & 17,0 & 16,4 & 16,4 & 16,5 \\
\hline Fibra bruta, \% MS & 15,1 & 14,9 & 14,6 & 14,7 \\
\hline Cenizas, \% MS & 7,6 & 7,5 & 7,5 & 7,5 \\
\hline Fibra neutrodetergente, $\%$ MS & 28,2 & 27,5 & 27,0 & 26,9 \\
\hline Carbohidratos no fibrosos, \% MS & 44,8 & 43,0 & 43,6 & 43,3 \\
\hline Almidón, \% MS & 34,5 & 33,9 & 33,5 & 33,3 \\
\hline Grasa (hidrólisis ácida), \% MS & 2,4 & 5,6 & 5,5 & 5,8 \\
\hline \multicolumn{5}{|c|}{ Ácidos grasos aportados por el aceite, g/d } \\
\hline C16:0 & - & 1,8 & 2,9 & 2,6 \\
\hline C18:0 & - & 1,4 & 2,0 & 1,8 \\
\hline $\mathrm{C} 18: 1 \mathrm{c} 9$ & - & 41,0 & 14,2 & 10,0 \\
\hline $\mathrm{C} 18: 2 \mathrm{c} 9 \mathrm{c} 12$ & - & 2,7 & 27,9 & 8,0 \\
\hline $\mathrm{C} 18: 3 \mathrm{c} 9 \mathrm{c} 12 \mathrm{c} 15$ & - & - & - & 23,9 \\
\hline
\end{tabular}

${ }^{1} \mathrm{GAO}$, GN y LIN: dietas enriquecidas con aceite de girasol alto oleico, girasol normal y lino, respectivamente. ${ }^{2}$ La dieta CONTROL aportó $5,1,0,8$, $5,5,14,4$ y $3,4 \mathrm{~g} / \mathrm{d}$ de 16:0, 18:0, 18:1c9, C18:2c9c12 y C18:3c9c12c15, respectivamente, calculado de acuerdo con INRA (2002). ${ }^{3}$ Incluido en el concentrado respectivo.

${ }^{1} \mathrm{GAO}$, GN and LIN: diets enriched with high oleic sunflower oil, regular sunflower oil and linseed oil, respectively. ${ }^{2} \mathrm{CONTROL}$ supplied 5.1, 0.8, 5.5, 14.4 and $3.4 \mathrm{~g} / \mathrm{d}$ of 16:0, 18:0, 18:1c9, C18:2c9c12 and C18:3c9c12c15, respectively, calculated according to INRA (2002). ${ }^{3}$ Included in the respective concentrate.

\section{ANÁLISIS ESTADÍSTICO}

Se utilizó un modelo completamente al azar con una covariable. El estudio estadístico se realizó con el procedimiento GENMOD de SAS 9.1 (SAS Inst Inc, Cary, NC) según el modelo: $Y i j=\mu+\alpha i+\beta X i j+\varepsilon i j$, donde, Yij $=$ variable dependiente; $\mu=$ media general $\alpha \mathrm{i}=$ efecto del tratamiento ( $\mathrm{i}=1$ a 4 ); $\beta \mathrm{Xij}=$ covariable (valor preexperimental de la variable dependiente); $\varepsilon \mathrm{ij}=$ error residual. $\mathrm{La}$ comparación de las medias de mínimos cuadrados se realizó por el procedimiento de la menor diferencia significativa. El nivel de significación se estableció en $\mathrm{P}<0,05$.

\section{RESULTADOS}

Las dietas con aceite añadido aportaron a los animales una cantidad de AGI claramente mayor que la dieta control y el grado de insaturación fue apreciablemente diferente entre ellas. El ácido oleico representó casi el $50 \%$ de los ácidos grasos totales aportado por la dieta GAO. El ácido linoleico representó en torno al $40 \%$ de los ácidos grasos totales aportados por la dieta GN, duplicando el contenido de ácido oleico. En la dieta LIN, el aporte de ácido $\alpha$-linolénico fue más del doble del de los ácidos oleico o linoleico (cuadro 1).

El contenido de ácidos grasos de la grasa láctea se muestra en el cuadro 2. Se identificaron y cuantificaron un total de 72 ácidos grasos. Los AGS de cadena corta y media mostraron respuestas comunes a los tres aceites añadidos. El contenido de C4:0, C6:0 y C8:0 de la grasa láctea no disminuyó por la adición de aceite a la dieta basal, excepto C8:0 en el tratamiento GAO $(\mathrm{P}<0,05)$. El contenido de $\mathrm{C} 10: 0 \mathrm{y}$ C12:0 fue numéricamente inferior en los tratamientos con aceite en comparación con el CONTROL pero las diferencias solamente fueron significativas $(\mathrm{P}<0,05)$ para el contenido de C10:0 en GAO y de C12:0 en GN. Por el contrario, 
Cuadro 2. Perfil de ácidos grasos de la grasa láctea ( $\mathrm{g} / 100 \mathrm{~g}$ de ésteres metílicos de ácidos grasos totales) de cabras que consumieron una dieta basal sin aceite añadido (CONTROL) o adicionada con $48 \mathrm{~g} / \mathrm{d}$ de aceite de girasol alto oleico (GAO), aceite de girasol normal (GN) o aceite de lino (LIN).

Milk fat fatty acid profile ( $\mathrm{g} / 100 \mathrm{~g}$ of total fatty acid methyl esters) of dairy goats fed diets added none oil (CONTROL), or $48 \mathrm{~g} / \mathrm{d}$ of high oleic sunflower oil (GAO), regular sunflower oil (GN) or linseed oil (LIN).

\begin{tabular}{|c|c|c|c|c|c|}
\hline \multirow[t]{2}{*}{ Ácidos grasos } & \multicolumn{4}{|c|}{ Tratamientos } & \multirow{2}{*}{$\mathrm{EE}$} \\
\hline & CONTROL & GAO & GN & LIN & \\
\hline \multicolumn{6}{|l|}{ Saturados } \\
\hline $\mathrm{C} 4: 0$ & $2,690^{\mathrm{b}}$ & $2,952^{\mathrm{ab}}$ & $3,243^{a}$ & $2,758^{\mathrm{b}}$ & 0,065 \\
\hline C6:0 & $2,998^{\mathrm{c}}$ & $2,970^{c}$ & $3,700^{\mathrm{a}}$ & $3,343^{b}$ & 0,059 \\
\hline $\mathrm{C} 8: 0$ & $3,257^{\mathrm{b}}$ & $2,852^{\mathrm{c}}$ & $4,091^{\mathrm{a}}$ & $3,899^{\mathrm{a}}$ & 0,065 \\
\hline C10:0 & $11,598^{\mathrm{a}}$ & $10,115^{\mathrm{b}}$ & $10,761^{\mathrm{ab}}$ & $11,407^{\mathrm{ab}}$ & 0,169 \\
\hline C12:0 & $4,618^{\mathrm{a}}$ & $4,101^{\mathrm{a}}$ & $3,004^{\mathrm{b}}$ & $4,417^{\mathrm{a}}$ & 0,121 \\
\hline C14:0 & $9,724^{a}$ & $8,823^{\mathrm{ab}}$ & $8,056^{\mathrm{b}}$ & $8,287^{b}$ & 0,181 \\
\hline C16:0 & $31,883^{\mathrm{a}}$ & $25,738^{\mathrm{b}}$ & $22,334^{c}$ & $23,385^{\mathrm{bc}}$ & 0,383 \\
\hline C18:0 & $7,258^{b}$ & $11,725^{\mathrm{a}}$ & $8,631^{\mathrm{b}}$ & $8,117^{\mathrm{b}}$ & 0,392 \\
\hline Otros saturados ${ }^{1}$ & $2,189^{b}$ & $2,035^{\mathrm{b}}$ & $2,093^{b}$ & $2,731^{\mathrm{a}}$ & 0,057 \\
\hline \multicolumn{6}{|l|}{ Monoinsaturados } \\
\hline C14:1c9 & $0,137^{\mathrm{ab}}$ & $0,106^{\mathrm{ab}}$ & $0,079^{\mathrm{b}}$ & $0,151^{\mathrm{a}}$ & 0,010 \\
\hline $\mathrm{C} 18: 1 \mathrm{c} 9$ & $15,355^{\mathrm{b}}$ & $20,535^{\mathrm{a}}$ & $16,327^{\mathrm{b}}$ & $14,890^{\mathrm{b}}$ & 0,280 \\
\hline C18:1t10 & $0,369^{b}$ & $0,237^{\mathrm{b}}$ & $1,341^{\mathrm{a}}$ & $0,331^{\mathrm{b}}$ & 0,073 \\
\hline C18:1t11 & $1,001^{\mathrm{c}}$ & $1,024^{c}$ & $5,428^{\mathrm{a}}$ & $3,533^{\mathrm{b}}$ & 0,201 \\
\hline C18:1t12 & $0,171^{\mathrm{b}}$ & $0,132^{\mathrm{b}}$ & $0,423^{\mathrm{a}}$ & $0,522^{\mathrm{a}}$ & 0,022 \\
\hline C18:1c12 & $0,165^{\mathrm{b}}$ & $0,041^{\mathrm{b}}$ & $0,585^{\mathrm{a}}$ & $0,886^{\mathrm{a}}$ & 0,060 \\
\hline $\mathrm{C} 18: 1 \mathrm{t} 15(+\mathrm{c} 11)$ & $0,255^{\mathrm{b}}$ & $0,300^{\mathrm{b}}$ & $0,334^{\mathrm{b}}$ & $0,612^{\mathrm{a}}$ & 0,017 \\
\hline C18:1c15 & $0,092^{\mathrm{b}}$ & $0,033^{b}$ & $0,069^{b}$ & $0,287^{\mathrm{a}}$ & 0,011 \\
\hline Otros monoinsaturados ${ }^{2}$ & $3,248^{\mathrm{b}}$ & $3,040^{\mathrm{b}}$ & $3,705^{\mathrm{a}}$ & $3,954^{\mathrm{a}}$ & 0,061 \\
\hline \multicolumn{6}{|l|}{ Poliinsaturados } \\
\hline $\mathrm{C} 18: 2 \mathrm{c} 9 \mathrm{c} 12$ & $1,825^{\mathrm{b}}$ & $1,190^{c}$ & $2,964^{\mathrm{a}}$ & $2,261^{\mathrm{b}}$ & 0,093 \\
\hline $\mathrm{C} 18: 2 \mathrm{c} 9 \mathrm{t} 11$ & $0,508^{b}$ & $0,420^{\mathrm{b}}$ & $2,225^{\mathrm{a}}$ & $1,625^{\mathrm{a}}$ & 0,105 \\
\hline $\mathrm{C} 18: 2 \mathrm{c} 9 \mathrm{t} 12$ & 0,029 & 0,020 & 0,023 & 0,035 & 0,002 \\
\hline $\mathrm{C} 18: 2 \mathrm{t} 11 \mathrm{c} 15$ & $0,041^{\mathrm{c}}$ & $0,035^{\mathrm{c}}$ & $0,134^{\mathrm{b}}$ & $0,856^{\mathrm{a}}$ & 0,024 \\
\hline $\mathrm{C} 18: 2 \mathrm{t} 11 \mathrm{t} 15$ & $0,014^{b}$ & $0,013^{b}$ & $0,021^{\mathrm{ab}}$ & $0,025^{\mathrm{a}}$ & 0,002 \\
\hline Otros diinsaturados ${ }^{3}$ & $0,257^{\mathrm{c}}$ & $0,224^{c}$ & $0,392^{\mathrm{b}}$ & $0,632^{\mathrm{a}}$ & 0,011 \\
\hline $\mathrm{C} 18: 3 \mathrm{c} 9 \mathrm{c} 12 \mathrm{c} 15$ & $0,211^{\mathrm{b}}$ & $0,134^{\mathrm{b}}$ & $0,220^{\mathrm{b}}$ & $0,778^{\mathrm{a}}$ & 0,033 \\
\hline $\mathrm{C} 18: 3 \mathrm{c} 9 \mathrm{t} 11 \mathrm{c} 15$ & $0,028^{b}$ & $0,036^{\mathrm{b}}$ & $0,037^{b}$ & $0,103^{\mathrm{a}}$ & 0,048 \\
\hline $\mathrm{C} 18: 3 \mathrm{c} 9 \mathrm{t} 11 \mathrm{t} 15$ & $\mathrm{Nd}$ & $\mathrm{Nd}$ & $\mathrm{Nd}$ & 0,039 & 0,006 \\
\hline Otros poliinsaturados ${ }^{4}$ & $0,268^{b}$ & $0,235^{\mathrm{b}}$ & $0,348^{\mathrm{a}}$ & $0,243^{\mathrm{b}}$ & 0,010 \\
\hline \multicolumn{6}{|l|}{ Sumatorios } \\
\hline ESaturados & $76,072^{\mathrm{a}}$ & $72,083^{\mathrm{b}}$ & $65,705^{\mathrm{c}}$ & $67,922^{\mathrm{c}}$ & 0,302 \\
\hline ¿Monoinsaturados & $20,898^{c}$ & $25,395^{\mathrm{b}}$ & $28,069^{\mathrm{a}}$ & $25,335^{\mathrm{b}}$ & 0,196 \\
\hline ¿Poliinsaturados & $3,594^{\mathrm{b}}$ & $2,159^{c}$ & $6,107^{\mathrm{a}}$ & $6,660^{\mathrm{a}}$ & 0,169 \\
\hline$\Sigma \mathrm{C} 18: 1 \mathrm{t}$ & $2,213^{c}$ & $2,333^{\mathrm{c}}$ & $8,933^{\mathrm{a}}$ & $5,991^{\mathrm{b}}$ & 0,207 \\
\hline
\end{tabular}


Cuadro 2. (continuación).

Relaciones

\begin{tabular}{lccccc} 
C14:1c9/C14:0+C14:1c9 & $0,014^{\mathrm{ab}}$ & $0,010^{\mathrm{b}}$ & $0,011^{\mathrm{ab}}$ & $0,017^{\mathrm{a}}$ & 0,001 \\
C18:2c9t12/C18:1t12+C18:2c9t12 & $0,142^{\mathrm{a}}$ & $0,140^{\mathrm{a}}$ & $0,056^{\mathrm{b}}$ & $0,066^{\mathrm{b}}$ & 0,008 \\
C18:2c9c12/C18:1c12+C18:2c9c12 & $0,917^{\mathrm{a}}$ & $0,950^{\mathrm{a}}$ & $0,839^{\mathrm{b}}$ & $0,733^{\mathrm{c}}$ & 0,007 \\
C18:3c9t11c15/C18:2t11c15+C18:3c9t11c15 & $0,455^{\mathrm{a}}$ & $0,475^{\mathrm{a}}$ & $0,227^{\mathrm{b}}$ & $0,090^{\mathrm{c}}$ & 0,013 \\
C18:2c9c12/C18:3c9c12c15 & $10,932^{\mathrm{b}}$ & $9,448^{\mathrm{b}}$ & $15,506^{\mathrm{a}}$ & $2,631^{\mathrm{c}}$ & 0,404 \\
Índice de aterogenicidad $^{5}$ & $3,178^{\mathrm{a}}$ & $2,331^{\mathrm{b}}$ & $1,636^{\mathrm{c}}$ & $1,950^{\mathrm{c}}$ & 0,044 \\
\hline
\end{tabular}

a,b,c En una misma fila, las medias sin un superíndice común difieren significativamente $(\mathrm{P}<0,05)$.

${ }^{1}$ Suma de C5:0, C7:0, 4-metiloctanoato, C9:0, metildecanoato, metildodecanoato, C13:0 iso, C13:0 anteiso, C14:0 iso, metiltetradecanoato, C15:0 iso, C15:0 anteiso, C15:0, C16:0 iso, C17:0, C19:0, C20:0, C21:0, C22:0 y ácido cetoesteárico. ${ }^{2}$ Suma de C10:1c9(+C11:0+C12:0 iso), C12:1c9(+C13:0), C15:1c9, C16:1t8, C16:1t9(+C17:0 iso), C16:1c7, C16:1c8, C16:1c9(+C17:0 anteiso), C16:1c13, C17:1c9, C18:1t6(+t7+t8), C18:1t9, C18:1c13, C18:1t16(+c14), C18:1c16 y C20:1c11. ${ }^{3}$ Suma de C18:2t9t12(+c9t13+t8c12), C18:2t8c13, C18:2t9c12, C18:2t12t14, C18:2t11t13 y C18:2 no identificados. ${ }^{4}$ Suma de C18:3c6c9c12, C20:4n-6, C20:5n-3, C22:4n-6, C22:5n-3 y C22:6n-3. ${ }^{5}(\mathrm{C} 12: 0+4 \times \mathrm{C} 14: 0+\mathrm{C} 16: 0) /(\mathrm{AGMI}+\mathrm{AGPI})$, de acuerdo con Ulbricht y Southgate (1991). Nd: No detectado.

a,b,c In a row, means without a common superscript are significantly different $(\mathrm{P}<0.05)$.

${ }^{1}$ Sum of C5:0, C7:0, 4-methyloctanoate, C9:0, methyldecanoate, methyldodecanoate, C13:0 iso, C13:0 anteiso, C14:0 iso, methyltetradecanoate, C15:0 iso, $\mathrm{C} 15: 0$ anteiso, $\mathrm{C} 15: 0, \mathrm{C} 16: 0$ iso, $\mathrm{C} 17: 0, \mathrm{C} 19: 0, \mathrm{C} 20: 0, \mathrm{C} 21: 0, \mathrm{C} 22: 0$ and 10-keto-18:0. ${ }^{2} \mathrm{Sum}$ of $\mathrm{C} 10: 1 \mathrm{c} 9(+\mathrm{C} 11: 0+\mathrm{C} 12: 0$ iso $), \mathrm{C} 12: 1 \mathrm{c} 9(+\mathrm{C} 13: 0)$, C15:1c9, C16:1t8, C16:1t9(+C17:0 iso), C16:1c7, C16:1c8, C16:1c9(+C17:0 anteiso), C16:1c13, C17:1c9, C18:1t6(+t7+t8), C18:1t9, C18:1c13, $\mathrm{C} 18: 1 \mathrm{t} 16(+\mathrm{c} 14), \mathrm{C} 18: 1 \mathrm{c} 16$ and $\mathrm{C} 20: 1 \mathrm{c} 11 .{ }^{3} \mathrm{Sum}$ of $\mathrm{C} 18: 2 \mathrm{t} 9 \mathrm{t} 12(+\mathrm{c} 9 \mathrm{t} 13+\mathrm{t} 8 \mathrm{c} 12), \mathrm{C} 18: 2 \mathrm{t} 8 \mathrm{c} 13, \mathrm{C} 18: 2 \mathrm{t} 9 \mathrm{c} 12, \mathrm{C} 18: 2 \mathrm{t} 12 \mathrm{t} 14, \mathrm{C} 18: 2 \mathrm{t} 11 \mathrm{t} 13$ and other unidentified C18:2. ${ }^{4}$ Sum of C18:3c6c9c12, C20:4n-6, C20:5n-3, C22:4n-6, C22:5n-3 and C22:6n-3. ${ }^{5}(\mathrm{C} 12: 0+4 \times \mathrm{C} 14: 0+\mathrm{C} 16: 0) /(\mathrm{AGMI}+\mathrm{AGPI})$, according to Ulbricht y Southgate (1991). Nd: Not detected.

el contenido de C14:0 y C16:0 disminuyó significativamente $(\mathrm{P}<0,05)$ en la grasa láctea de todos los tratamientos con aceite, alcanzando valores que fueron por término medio 14 y $25 \%$ inferiores, respectivamente, a los del CONTROL.

Los ácidos grasos de 18 átomos de carbono mostraron respuestas características a cada uno de los aceites añadidos. El contenido de los ácidos esteárico (C18:0) y oleico fue significativamente mayor $(\mathrm{P}<0,05)$ en la grasa láctea del tratamiento GAO, mientras que los niveles de C18:1t10 y C18:2c9c12 fueron más elevados $(\mathrm{P}<0,05)$ en GN. Por otra parte, los mayores porcentajes de C18:1t15(+c11), C18:1c15, C18:2t11c15, C18:2t11t15, C18:3c9c12c15, C18:3c9t11c15 y C18:3c9t11t15 se observaron en el tratamiento LIN $(\mathrm{P}<0,05)$. También se observaron respuestas características comunes a los aceites de girasol normal y lino: los contenidos de C18:1t11, C18:1t12, C18:1c12 y C18:2c9t11 fueron significativamente mayores $(\mathrm{P}<0,05)$ en los tratamientos GN y LIN comparados con CONTROL y GAO, que fueron iguales entre sí $(\mathrm{P}>0,05)$. El contenido de $\mathrm{C} 18: 1 \mathrm{t} 11$ fue significativamente mayor $(\mathrm{P}<0,05)$ en GN que en LIN pero no hubo diferencias significativas entre ambos tratamientos en el contenido de C18:2c9t11 ( $\mathrm{P}>0,05)$ aunque el aumento en relación con el CONTROL fue mayor en GN (338\%) que en LIN (220\%).

Los AGS y los ácidos grasos monoinsaturados (AGMI) respondieron de forma común a la adición de los tres aceites: disminución significativa $(\mathrm{P}<0,05)$ del primer grupo y aumento significativo $(\mathrm{P}<0,05)$ del segundo. Los contenidos de AGPI y AGMI trans de 18 átomos de carbono mostraron un aumento significativo $(\mathrm{P}<0,05)$ en los tratamientos GN y LIN, respuesta que no se observó en GAO.
Respecto a la relación C14:1c9/C14:0 + C14:1c9, que se utiliza habitualmente como índice de la actividad de la enzima delta-9 desaturasa mamaria, fue significativamente menor $(\mathrm{P}<0,05)$ en GAO y GN, mientras que las relaciones C18:2c9t12/C18:1t12 + C18:2c9t12 y C18:2c9c12/ C18:1c12 + C18:2c9c12 fueron menores en GN y LIN. La relación C18:3c9t11c15/C18:2t11c15 + C18:3c9t11c15 tuvo el valor menor $(\mathrm{P}<0,05)$ en LIN. La ratio linoleico/ $\alpha-$ linolénico fue significativamente menor en $\operatorname{LIN}(\mathrm{P}<0,05)$, mayor en GN $(\mathrm{P}<0,05)$ y no cambió en GAO $(\mathrm{P}>0,05)$ en comparación con el tratamiento CONTROL. La adición de aceite de girasol normal a la dieta aumentó un $42 \%$ el valor de la ratio mientras que la adición de aceite de lino lo redujo un $76 \%$ en comparación con la dieta basal. $\mathrm{El}$ índice de aterogenicidad fue significativamente menor $(\mathrm{P}<0,05)$ en todos los tratamientos con aceite en comparación con el CONTROL.

\section{DISCUSIÓN}

La reducción del contenido de AGS de cadena media de la grasa láctea observada en los tratamientos con aceite podría atribuirse a un déficit de sustratos en la glándula mamaria para la síntesis de novo por un efecto negativo de los AGI sobre la digestión ruminal. Sin embargo, las pequeñas diferencias de la proporción ruminal de acetato entre tratamientos 651, 651, 660 y $646 \mathrm{mmol} / 1000$ mmol de ácidos grasos volátiles en CONTROL, GAO, GN y LIN, respectivamente, de acuerdo con la ecuación de Vlaeminck y col (2006) y la ausencia de efectos negativos sobre el contenido de AGS de cadena corta, que 
también son sintetizados en la ubre, no avalarían la hipótesis de una falta de sustratos para la síntesis de novo en las cabras que consumieron las dietas con aceite añadido. Es más probable que la mayor disponibilidad de ácidos grasos de cadena larga para la ubre en los tratamientos con aceite haya tenido un efecto negativo sobre la ratio de las actividades de las enzimas acetil-CoA carboxilasa y ácido graso sintetasa, que participan en la síntesis de novo (Bernard y col 2009 ${ }^{\mathrm{b}}$ ). En el mismo sentido, el hecho de que el contenido de AGS de cadena corta no se afectara negativamente coincide con los resultados de investigaciones in vitro citados por Bauman y Davis (1974) que muestran que la reducción de la ratio de las actividades de las enzimas acetil-CoA carboxilasa y ácido graso sintetasa en las células mamarias cambia el patrón de ácidos grasos sintetizados hacia los de cadena más corta.

El bajo contenido de los ácidos esteárico y oleico en la grasa láctea del tratamiento CONTROL podría explicarse por el menor aporte de ácidos grasos de 18 átomos de carbono de dicha dieta a los animales (cuadro 1). El ácido oleico de la grasa láctea puede proceder del ácido oleico de la dieta o de la grasa corporal movilizada o bien puede formarse en la ubre por delta-9 desaturación del ácido esteárico captado, procedente a su vez de la movilización de reservas grasas o de la biohidrogenación (BH) ruminal de los AGI de 18 átomos de carbono de la dieta. Enjalbert y col (1998) encontraron que aproximadamente la mitad del C18:0 captado por la glándula mamaria era desaturado a $\mathrm{C} 18: 1 \mathrm{c} 9$ en vacas. El origen endógeno de los ácidos esteárico y oleico puede descartarse en el presente trabajo porque las cabras no perdieron peso (Martínez Marín y col 2012 ${ }^{\text {b }}$ y la transferencia aparente de ambos en relación con los ácidos grasos de 18 átomos de carbono consumidos fue inferior a $60 \%$ en las dietas con aceite añadido. Por otra parte, la ausencia de un incremento de los ácidos esteárico y oleico y el mayor contenido de AGMI trans de 18 átomos de carbono y AGPI en la grasa láctea de los tratamientos GN y LIN indicaría que la $\mathrm{BH}$ ruminal de los AGI de 18 átomos de carbono fue menos completa en los animales que recibieron dichos tratamientos, con la consiguiente reducción del ácido esteárico disponible para su captación mamaria. Bernard y col (2005) encontraron mayor cantidad de C18:0 + C18:1c9 en la grasa láctea de cabras cuya dieta fue enriquecida con ácido oleico en vez de ácido $\alpha$-linolénico. Por otro lado, el menor índice de desaturación observado en GAO (cuadro 2) coincide con Bernard y col (2005) quienes observaron menores valores de actividad de la estearoil-CoA desaturasa láctea de las cabras cuando la dieta fue enriquecida con ácido oleico en comparación con la dieta control.

Es bien conocido que las dietas ricas en ácido linoleico y con una baja relación forraje/concentrado incrementan el contenido de C18:1t10 en la grasa láctea (Shingfield y Griinari 2007) debido a un cambio en las rutas de $\mathrm{BH}$ ruminal que resulta en mayor producción de C18:1t10 vía C18:2t10c12 (McKain y col 2010). La BH ruminal de C18:3n-3 da lugar a una menor formación de C18:1t10 que la del ácido linoleico (Jouany y col 2007). En esta línea, el mayor porcentaje de C18:1t10 se observó en la grasa láctea del tratamiento GN (cuadro 2) y no hubo diferencias significativas en los tratamientos GAO y LIN respecto al CONTROL. La ausencia de cambios del contenido de C18:1t10 de la grasa láctea en GAO contrasta con los resultados obtenidos por Ollier y col (2009) en cabras cuya dieta fue enriquecida con ácido oleico. Esta diferencia podría explicarse por una mayor $\mathrm{BH}$ y una menor isomerización del ácido oleico aportado en el presente estudio ya que C18:1t10 es el principal producto de la isomerización del ácido oleico por los microorganismos ruminales (AbuGhazaleh y col 2005).

El aumento del contenido de C18:1t11 en la grasa láctea de los tratamientos GN y LIN era esperado porque el ácido vaccénico es un intermediario común en las rutas de BH de los ácidos linoleico y $\alpha$-linolénico (Palmquist y col 2005). Datos obtenidos por Bernard y col $\left(2009^{\mathrm{a}}\right)$ indican que la adición a la dieta de cabras de cantidades iguales de aceite de girasol o aceite de lino resulta en un mayor incremento de C18:1t11 en la grasa láctea con el primero. Por otra parte, Ollier y col (2009) tampoco observaron cambios en el contenido de C18:1t11 de la grasa láctea cuando las cabras consumieron una dieta enriquecida con ácido oleico en comparación con la dieta control.

La desaturación en posición 9 de AGMI trans de 18 átomos de carbono en la ubre de vacas fue puesta de manifiesto por primera vez por Griinari y col (2000). En el presente trabajo, aparte del ácido ruménico, se identificaron dos ácidos grasos diinsaturados de 18 átomos de carbono con un doble enlace en posición c9: C18:2c9t12 y $\mathrm{C} 18: 2 \mathrm{c} 9 \mathrm{c} 12$. Ambos pudieron formarse en la ubre por delta-9 desaturación de los respectivos AGMI: C18:1t12 y C18:1c12. La delta-9 desaturación de C18:1t12 no ocurrió ya que su contenido fue mayor en GN y LIN mientras que los valores de la relación C18:2c9t12/C18:1t12 + C18:2c9t12 fueron menores en dichos tratamientos (cuadro 2). Por otro lado, la delta-9 desaturación de C18:1c12, si ocurrió, no fue cuantitativamente relevante porque la relación C18:2c9c12/C18:1c12 + C18:2c9c12 fue menor en LIN, que mostró un contenido mayor de C18:1c12 pero un nivel de ácido linoleico similar al tratamiento CONTROL (cuadro 2). Esto indica que todo o la mayor parte del ácido linoleico presente en la grasa láctea fue de origen dietético, escapado del rumen sin biohidrogenar.

Dado que el ácido ruménico no es un intermediario en la $\mathrm{BH}$ del ácido $\alpha$-linolénico, el incremento del contenido de ácido ruménico en la grasa láctea observado en el tratamiento LIN fue debido a una mayor producción de ácido vaccénico en el rumen, posteriormente desaturado en la glándula mamaria por la delta-9 desaturasa (Griinari y col 2000). Por otro lado, un mayor incremento del contenido de ácido ruménico en la grasa láctea cuando se suministran dietas enriquecidas con ácido linoleico en compara- 
ción con cantidades iguales de ácido $\alpha$-linolénico también se ha encontrado en otros trabajos (Bernard y col 2009a, Martínez Marín y col 2011).

La inclusión de fuentes de grasa ricas en ácido $\alpha$-linolénico en la dieta de rumiantes lecheros tiene como consecuencia un aumento de isómeros característicos en la grasa láctea. El grupo de isómeros C18:3c9t11c15, C18:2t11c15 y C18:1c15 corresponde a una ruta de BH ruminal propuesta por Harfoot y Hazlewood (1997) mientras que el grupo C18:3c9t11t15, C18:2t11t15 y C18:1t15 coincide con la vía postulada por Gómez-Cortés y col (2009). Ambos grupos de ácidos grasos fueron identificados en nuestro trabajo y aumentaron significativamente en el tratamiento LIN (cuadro 2). El aumento del pico cromatográfico que incluye los isómeros C18:1t15 y C18:1c11 fue debido probablemente al incremento del isómero C18:1t15 ya que C18:1c11 es de origen microbiano (Fulco 1983). El aumento del contenido del isómero C18:1c15 en la grasa láctea del tratamiento LIN también fue observado por Ollier y col (2009) en respuesta al consumo de fuentes de grasa ricas en C18:3n-3. Además del posible origen ruminal por isomerización del ácido $\alpha$-linolénico, los isómeros C18:3c9t11c15 y C18:3c9t11t15 presentes en la grasa láctea pueden originarse también por delta-9 desaturación de los respectivos ácidos grasos diinsaturados. El isómero C18:3c9t11c15 se ha encontrado en el plasma de vacas lecheras cuya dieta incluyó semilla de lino cruda o extrusionada (Akraim y col 2007) lo que indica un origen ruminal. Además, el bajo valor de la relación C18:3c9t11c15/C18:2t11c15 + C18:3c9t11c15 en el tratamiento LIN no apoya un papel relevante de la delta- 9 desaturasa mamaria en la generación de C18:2c9t11c15 (cuadro 2). De todo ello se deduce que la mayor parte del C18:3c9t11c15 tuvo su origen en el rumen, en coincidencia con los resultados de Martínez Marín y col (2011). Por otro lado, no pudo establecerse la posible delta- 9 desaturación mamaria de C18:2t11t15 para producir C18:3c9t11t15 porque el contenido de este último en la grasa láctea de los tratamientos CONTROL, GN y GAO estuvo por debajo de su límite de detección cromatográfico. Conviene destacar, sin embargo, que Martínez Marín y col (2011) señalaron el probable origen mamario de C18:3c9t11t15 sin excluir totalmente su formación el rumen.

El valor de la ratio linoleico/ $\alpha$-linolénico en LIN fue inferior a 4 mientras que los restantes tratamientos mostraron valores iguales o superiores a 9 . De acuerdo con Simopoulos (2008), valores inferiores a 4 en la grasa de la dieta humana se asocian con una reducción de la mortalidad causada por enfermedad cardiovascular y del riesgo de cáncer de mama y tiene efectos positivos en enfermos de cáncer de colon y artritis reumatoide. Los valores obtenidos en el presente trabajo están de acuerdo con los resultados de estudios previos (Bernard y col 2009ª, Martínez Marín y col 2011) y pueden explicarse por el aporte de ácidos grasos de las dietas de cada tratamiento: el tra- tamiento LIN fue el único que suministró una cantidad importante de ácido $\alpha$-linolénico a los animales, GN proporcionó la mayor cantidad de ácido linoleico, y GAO no cambió el aporte de ninguno de ambos ácidos grasos en comparación con el tratamiento CONTROL (cuadro 1).

Se concluye que con independencia de su grado de insaturación, los aceites incluidos en la dieta tuvieron dos efectos comunes claros sobre las proporciones de ácidos grasos de la grasa láctea: reducción de los ácidos grasos saturados de cadena media y aumento de los ácidos grasos de 18 átomos de carbono. A diferencia del aceite de girasol alto oleico, los aceites de girasol normal y lino aumentaron la proporción de ácidos grasos de origen ruminal en la grasa láctea debido a una BH más incompleta de los ácidos grasos mayoritarios (linoleico y $\alpha$-linolénico) que aportaron a la dieta. Algunos de los ácidos grasos diinsaturados y triinsaturados de 18 átomos de carbono con un doble enlace en posición c9 presentes en la grasa láctea pudieron originarse en la glándula mamaria pero se necesitarían más estudios para confirmar este supuesto. En comparación con la adición de los aceites de girasol alto oleico y girasol normal a la dieta, la adición de aceite de lino aumentó el contenido de los ácidos vaccénico y ruménico de la grasa láctea sin modificar el contenido de C18:1t10 y redujo el valor de la relación entre los ácidos linoleico y $\alpha$-linolénico. Desde el punto de vista de la salud humana, el aceite de lino tuvo los efectos más favorables sobre el perfil de ácidos grasos de la leche de cabra.

\section{REFERENCIAS}

AbuGhazaleh AA, MB Riley, EE Thies, TC Jenkins. 2005. Dilution rate and $\mathrm{pH}$ effects on the conversion of oleic acid to trans $\mathrm{C} 18: 1$ positional isomers in continuous culture. $J$ Dairy Sci 88, 4334-4341.

Akraim F, MC Nicot, P Juaneda, F Enjalbert. 2007. Conjugated linolenic acid (CLnA), conjugated linoleic acid (CLA) and other biohydrogenation intermediates in plasma and milk fat of cows fed raw or extruded linseed. Animal 1, 835-843.

Bauchart D, A Roy, S Lorenz, JM Chardigny, A Ferlay, D Gruffat, JL Sébédio, Y Chilliard, D Durand. 2007. Butters varying in trans 18:1 and cis-9, trans-11 conjugated linoleic acid modify plasma lipoproteins in the hypercholesterolemic rabbit. Lipids 42, 123-133.

Bauman DE, CL Davis. 1974. Biosynthesis of milk fat. In: Larson BL, Smith VR (eds). Lactation: A Comprehensive Treatise. Volume 2. Academic Press, New York, USA, Pp 31-75.

Benjamin S, F Spener. 2009. Conjugated linoleic acids as functional food: an insight into their health benefits. Nutr Metab 6, 36-48.

Bernard L, J Rouel, C Leroux, A Ferlay, Y Faulconnier, P Legrand, Y Chilliard. 2005. Mammary lipid metabolism and milk fatty acid secretion in alpine goats fed plant lipids. J Dairy Sci 88, 1478-1489.

Bernard L, KJ Shingfield, J Rouel, A Ferlay, Y Chilliard. 2009ª Effect of plant oils in the diet on performance and milk fatty acid composition in goats fed diets based on grass hay or maize silage. Br J Nutr 101, 213-224. 
Bernard L, M Bonnet, C Leroux, KJ Shingfield, Y Chilliard. $2009^{\mathrm{b}}$. Effect of sunflower-seed oil and linseed oil on tissue lipid metabolism, gene expression, and milk fatty acid secretion in Alpine goats fed maize silage-based diets. J Dairy Sci 92, 6083-6094.

Bouattour MA, R Casals, E Albanell, X Such, G Caja. 2008. Feeding soybean oil to dairy goats increases conjugated linoleic acid in milk. J Dairy Sci 91, 2399-2407.

Chilliard Y, F Glasser, A Ferlay, L Bernard, J Rouel, M Doreau. 2007. Diet, rumen biohydrogenation and nutritional quality of cow and goat milk fat. Eur J Lipid Sci Technol 109, 828-855.

Enjalbert F, MC Nicot, C Bayourthe, R Moncoulon. 1998. Duodenal infusions of palmitic, stearic or oleic acids differently affect mammary gland metabolism of fatty acids in lactating dairy cows. $J$ Nutr $128,1525-1532$.

FEDNA, Fundación Española para el Desarrollo de la Nutrición Animal. 2010. Normas Fedna para la Formulación de Piensos Compuestos. Fundación Española para el Desarrollo de la Nutrición Animal, Madrid, España.

Field CJ, HH Blewett, S Proctor, D Vine. 2009. Human health benefits of vaccenic acid. Appl Physiol Nutr Metab 34, 979-991.

Fulco AJ. 1983. Fatty acid metabolism in bacteria. Prog Lipid Res 22, 133-160.

Gómez-Cortés P, C Tyburczy, JT Brenna, M Juárez, MA De la Fuente. 2009. Characterization of cis-9 trans-11 trans-15 C18:3 in milk fat by GC and covalent adduct chemical ionization tandem MS. J Lipid Res 50, 2412-2420.

Griinari JM, BA Corl, SH Lacy, PY Chouinard, KVV Nurmela, DE Bauman. 2000. Conjugated linoleic acid is synthesized endogenously in lactating dairy cows by $\Delta 9$ desaturase. J Nutr 130, 2285-2291.

Harfoot CG, GP Hazlewood. 1997. Lipid metabolism in the rumen. In: Hobson PN (ed). The Rumen Microbial Ecosystem. Blackie Academic \& Professional, London, UK, Pp 382-426.

INRA, Institut National de la Recherche Agronomique. 2002. Tables de composition et de valeur nutritive des matières premières destinées aux animaux d'élevage. INRA Éditions, Paris, France.

ISO-IDF. 2002 ${ }^{\mathrm{a}}$. Milk fat-Preparation of fatty acid methyl esters. International Standard ISO 15884-IDF 182: 2002. International Dairy Federation, Brussels, Belgium.

ISO-IDF. 2002 ${ }^{\text {b }}$. Milk fat-Determination of the fatty acid composition by gas-liquid chromatography. International Standard ISO 15885-IDF 184: 2002. International Dairy Federation, Brussels, Belgium.

Jouany JP, B Lassalas, M Doreau, F Glasser. 2007. Dynamic features of the rumen metabolism of linoleic acid, linolenic acid and linseed oil measured in vitro. Lipids 42 , 351-360.

Luna L, A Bach, M Juárez, MA De la Fuente. 2008. Effect of a diet enriched in whole linseed and sunflower oil on goat milk fatty acid composition and conjugated linoleic acid isomer profile. J Dairy Sci 91, 20-28.

Luna P, M Juárez, MA De la Fuente. 2005. Validation of a rapid milk fat separation method to determine the fatty acid profile by gas chromatography. J Dairy Sci 88, 3377-3381.

Maia FJ, AF Branco, GF Mouro, SM Coneglian, GT Dos Santos, TF Minella, KC Guimarães. 2006. Inclusão de fontes de óleo na dieta de cabras em lactação: produção, composição e perfil dos ácidos graxos do leite. $R$ Bras Zootec 35, 1504-1513.

Martínez Marín AL, P Gómez-Cortés, AG Gómez Castro, M Juárez, LM Pérez Alba, M Pérez Hernández, MA De la Fuente. 2011. Animal performance and milk fatty acid profile of dairy goats fed diets with different unsaturated plant oils. J Dairy Sci 94, 5359-5368.

Martínez Marín AL, P Gómez-Cortés, G Gómez Castro, M Juárez, L Pérez Alba, M Pérez Hernández, MA De la Fuente. $2012^{\mathrm{a}}$. Effects of feeding increasing dietary levels of high oleic or regular sunflower or linseed oil on fatty acid profile of goat milk. J Dairy Sci 95, 1942-1955.

Martínez Marín AL, M Pérez Hernández, LM Pérez Alba, D Carrión Pardo, AG Gómez Castro. 2012 . Adición de aceites vegetales a la dieta de cabras lecheras: efecto sobre la digestibilidad y los resultados productivos. Arch Med Vet 44, 21-28.

McKain N, KJ Shingfield, RJ Wallace. 2010. Metabolism of conjugated linoleic acids and 18:1 fatty acids by ruminal bacteria: products and mechanisms. Microbiology 156, 579-588.

Mele M, A Serra, A Buccioni, G Conte, A Pollicardo, P Secchiari. 2008. Effect of soybean oil supplementation on milk fatty acid composition from Saanen goats fed diets with different forage:concentrate ratios. Ital J Anim Sci 7, 297-311.

Ollier S, C Leroux, A De la Foye, L Bernard, J Rouel, Y Chilliard. 2009. Whole intact rapeseeds or sunflower oil in high-forage or high-concentrate diets affects milk yield, milk composition, and mammary gene expression profile in goats. J Dairy Sci 92, 5544-5560.

Palmquist DL, AL Lock, KJ Shingfield, DE Bauman. 2005. Biosynthesis of conjugated linoleic acid in ruminants and humans. In: Taylor SL (ed). Advances in Food and Nutrition Research. Academic Press, New York, USA, Pp 179-217.

Shingfield KJ, JM Griinari. 2007. Role of biohydrogenation intermediates in milk fat depression. Eur J Lipid Sci Technol 109, 799-816.

Simopoulos AP. 2008. The importance of the omega-6/omega-3 fatty acid ratio in cardiovascular disease and other chronic diseases. Exp Biol Med 233, 674-688.

Ulbricht TLV, DAT Southgate. 1991. Coronary heart disease: Seven dietary factors. Lancet 338, 985-992.

Vlaeminck B, V Fievez, S Tamminga, RJ Dewhurst, A Van Vuuren, D De Brabander, D Demeyer. 2006. Milk oddand branched-chain fatty acids in relation to the rumen fermentation pattern. J Dairy Sci 89, 3954-3964. 